\title{
Exploring functional genomics for drug target and therapeutics discovery in Plasmodia
}

\author{
L. Birkholtz ${ }^{\mathrm{a}, \mathrm{b}}$, A.C. van Brummelen ${ }^{\mathrm{a}}$, K. Clark ${ }^{\mathrm{a}}$, J. Niemand ${ }^{\mathrm{a}}$, E. Maréchal ${ }^{\mathrm{c}}$, \\ M. Llinás ${ }^{\mathrm{d}}$ and A.I. Louw ${ }^{\mathrm{a}, \mathrm{b}}$
}

a Department of Biochemistry, University of Pretoria, Pretoria 0002, South Africa

b African Centre for Gene Technologies, University of Pretoria, Pretoria 0002, South Africa

c UMR 5168 CNRS-CEA-INRA-Université Joseph Fourier, Laboratoire de Physiologie Cellulaire Végétale, Institut de Recherches en Technologies et Sciences pour le Vivant, CEA Grenoble, 17 rue des Martyrs, F-38054, Grenoble cedex 09, France

d Department of Molecular Biology, Lewis-Sigler Institute for Integrative Genomics, Princeton University, 246 Carl Icahn Laboratory, Princeton, NJ 08544, USA

\begin{abstract}
Functional genomics approaches are indispensable tools in the drug discovery arena and have recently attained increased attention in antibacterial drug discovery research. However, the application of functional genomics to post-genomics research of Plasmodia is still in comparatively early stages. Nonetheless, with this genus having the most species sequenced of any eukaryotic organism so far, the Plasmodia could provide unique opportunities for the study of intracellular eukaryotic pathogens. This review presents the status quo of functional genomics of the malaria parasite including descriptions of the transcriptome, proteome and interactome. We provide examples for the in silico mining of the $\mathrm{X}$-ome data sets and illustrate how $\mathrm{X}$-omic data from drug challenged parasites might be used in elucidating amongst others, the mode-of-action of inhibitory compounds, validate potential targets and discover novel targets/therapeutics.
\end{abstract}

\begin{abstract}
Abbreviations: IDC, intraerythrocytic developmental cycle; MOA, mode-of-action; MudPIT, multidimensional protein identification technology; GO, gene ontology; SAGE, serial analysis of gene expression; ICAT, isotopically coded affinity tags; iTRAQ, isotope tags for relative and absolute quantification
\end{abstract}

\section{Article Outline}

1. Introduction

2. The Plasmodium transcriptome

3. The Plasmodium proteome 
4. Integrative biology from the Plasmodium transcriptome and proteome

5. The Plasmodium interactome

6. In silico navigation of the Plasmodium X-omes

7. Plasmodium $\mathrm{X}$-omes in drug target and therapeutics discovery

7.1. Understanding inhibitory compounds' MOA through transcriptomics

7.2. Proteome-drug interactions

7.3. Interactome as drug target in malaria

7.4. Validation of drug targets

8. Conclusions

Acknowledgements

References

\section{Introduction}

With 300-500 million clinical cases and about 3 million deaths annually, especially amongst children less than 5 years of age, malaria is one of the world's most important tropical infectious diseases (Guerra et al., 2006). Combating malaria requires significant financial and organizational resources, yet malaria itself restrains economic development, creating a vicious circle in developing countries ([Pink et al., 2005] and [Sachs and Malaney, 2002]). The devastating socio-economic and public health impact of malaria is mostly experienced in sub-Saharan Africa and is galvanized by the emergence and rapid spread of drug-resistant parasites and the lack of a licensed vaccine. Plasmodium falciparum, one of the four known human Plasmodium species, is the most lethal and the principal cause of the morbidity and mortality in sub-Saharan Africa.

The discovery of new and robust antimalarial drugs, as well as new targets, can be expedited by including global mapping of pharmacological space and the introduction of functional and structural genomics, proteomics, metabolomics and bioinformatics at the very early stages of the discovery pipeline ([Paolini et al., 2006] and [Sawyer, 2006]). Functional genomics has become an indispensable tool in the drug discovery process ([Ohlstein et al., 2000] and [Wang et al., 2004]) due to its capacity to monitor the effects of perturbations at a global level, as opposed to the molecular level of the more traditional methods. Antibacterial drug discovery has benefited greatly by the application of functional genomics by improving knowledge of gene function, bacterial physiology, effects of antibiotics on bacterial metabolism, in vitro target identification and the modeof-action of novel antibacterial agents (Freiberg and Brotz-Oesterhelt, 2005).

The application of X-omics sciences in antimalarial drug discovery is by comparison only at the initial stages of exploration but is expected to allow deciphering of the transcriptome, proteome and metabolome responses of the parasite to environmental perturbations, and integration of data to reveal the effects on the biological network structure (Freiberg and Brotz-Oesterhelt, 2005). X-omics data provide comprehensive information to, amongst others, understand the mode-of-action (MOA) of inhibitory compounds, allow lead optimization, validate drug targets (chemical validation strategies), identify drug targets by identifying families of genes/gene products that are 
more amenable as drug targets ('druggable genes'), guilt-by-association functional annotation of hypothetical proteins and specialized gene expression regulation systems (Box 1).

Box 1. Applications of functional genomics strategies to drug targets and therapeutics discovery in the malaria parasite

\begin{tabular}{|c|c|}
\hline Genome-wide questions & $\begin{array}{l}\text { Transcriptome-specific } \\
\text { questions }\end{array}$ \\
\hline Lifecycle development (stage-specific expression) & Transcriptional machinery \\
\hline Reproduction genes (strategy-specific expression) & Regulation of transcription \\
\hline Drug resistance mechanisms & Transcriptional inheritance \\
\hline \multicolumn{2}{|l|}{ Mechanism of drug action } \\
\hline Reponses to environmental stressors & Proteome-specific questions \\
\hline Drug target specification & Post-transcriptional regulation \\
\hline Host-specific adaptation and expression & Post-translational repression \\
\hline \multicolumn{2}{|l|}{ Identification of vaccine targets } \\
\hline Virulence determinants & Interactome-specfic questions \\
\hline \multirow[t]{3}{*}{ Severe disease progression in vivo } & Protein function \\
\hline & Relationships \\
\hline & Regulatory mechanisms \\
\hline \multicolumn{2}{|l|}{ Biological and mechanistic insights } \\
\hline Metabolic pathways & \\
\hline \multicolumn{2}{|l|}{ Identity determination of hypothetical proteins } \\
\hline \multicolumn{2}{|l|}{ Cell cycle regulators } \\
\hline \multicolumn{2}{|l|}{ Sex determinants } \\
\hline \multicolumn{2}{|l|}{ Chemical validation of drug targets } \\
\hline \multicolumn{2}{|l|}{ Mode-of-action of inhibitory compounds } \\
\hline \multicolumn{2}{|l|}{ Improved drug target action } \\
\hline $\begin{array}{c}\text { Gene expression regulators (transcription and } \\
\text { translation) }\end{array}$ & \\
\hline
\end{tabular}




\begin{tabular}{|c|}
\hline Virulence factors \\
\hline Specialised organelle function and metabolism \\
\cline { 1 - 2 } Damage compensation \\
\hline
\end{tabular}

This review provides an overview of the current state of X-omics (specifically transcriptomics, proteomics and interactomics) of Plasmodia, with a special focus on the potential applications thereof to malarial drug target and therapeutics discovery.

\section{The Plasmodium transcriptome}

Post-genome transcriptome analysis of $P$. falciparum was initiated on a whole genome scale by two concurrent DNA microarray-based descriptions in 2003, which monitored transcriptome changes during the lifecycle of the parasite. Most of the lifecycle stages were covered including mosquito-infective sporozoites, erythrocyte-invasive merozoites, six synchronized stages during the intraerythrocytic development cycle (IDC) and finally sexual gametocyte forms (Le Roch et al., 2003). A detailed investigation of the asexual IDC was performed by Bozdech et al. (2003), delineating this $48 \mathrm{~h}$ disease causing cycle over $1 \mathrm{~h}$ intervals. Although different technologies and approaches were used by both groups, the overall conclusions were similar. The active transcription of the majority $(>80 \%)$ of the predicted genes indicated a degree of overlap in the metabolic requirements during the different developmental stages of the parasite. However, $20 \%$ of all the predicted transcripts are IDC-specific and the high resolution of the Bozdech et al. (2003) study indicated a periodic, 'just-in-time' nature of transcript production for $>80 \%$ of these IDC transcripts. Genes are activated only once in a cascade manner, with peak expression just prior to the point at which their biological function is required (Bozdech et al., 2003).

Comparative transcriptomics of three geographically and phenotypically distinct strains of P. falciparum (3D7, Hb3, Dd2) characterized inter-strain differences (Llinás et al., 2006) but remarkably, a high level of conservancy was found between these strains with significant transcriptional differences limited to genes encoding surface antigens. These results indicated a simple, highly conserved and streamlined cascade of gene expression. More specialized reports described transcriptome analysis of the early stages of gametocytogenesis (Silvestrini et al., 2005) and transcriptional features involved in the mechanism of var gene silencing, which influenced regulation of genes distal to the var loci (Ralph et al., 2005). Comparison of wild type 3D7 P. falciparum to a gametocyte deficient clone with a gene-specific custom array revealed differential expression of transcription factors and kinases that may play a role in the commitment to gametocytogenesis, the modulation of which may be used to block early gametocytogenesis and ultimately transmission (Gissot et al., 2004). 
To understand the underlying biology of the parasite while residing in the host environment, two studies described in vivo transcriptional profiling of $P$. falciparum. The high degree of correlation between the in vitro and in vivo transcriptomes was highlighted but overexpression of genes encoding a sexual stage antigen as well as gene families that encode surface proteins was shown (Daily et al., 2004, Daily et al., 2005). Transcriptome analysis has also been utilized to understand the hosts' response to malaria infection. The response of Rhesus monkeys to $P$. cynomolgi bastionella infection was investigated using a human oligonucleotide chip (Ylostalo et al., 2005) whereas a mouse oligonucleotide chip was used to investigate the effects of $P$. berghei infection (Sexton et al., 2004). These studies showed that the expression of genes required for glycolysis, the host immune response and erythropoiesis are modified, which correlates with the underlying pathology of disease progression.

\section{The Plasmodium proteome}

Transcriptomics is at present the most comprehensive, high-throughput methodology by which immense amounts of data can be obtained on a global level but the results do not allow unambiguous extrapolations to protein levels or activities, metabolite composition or flux, etc. Proteomic studies are thus essential to probe mechanistic changes and explain global protein expression profiles, differential protein expression, posttranscriptional control, post-translational regulation and modifications, alternative splicing and processing, subcellular localization, host-pathogen interactions and moreover, the interconnectivity between these processes (Box 1).

Fewer reports are available of the parasite's proteome than of the transcriptome due to technical constraints. The most comprehensive study to date of the $P$. falciparum life cycle proteome provided information of $46 \%$ (2400 proteins) of all possible gene products using multidimensional protein identification technology (MudPIT). Proteins that are unique to only one developmental stage were identified with only 6\% (124 proteins) common to all four stages (sporozoites, merozoites, trophozoites and gametocytes) (Florens et al., 2002). These commonly shared proteins were mostly housekeeping proteins, including ribosomal and cytoskeletal proteins, transcription factors and histones. These results disagreed with those of Le Roch et al. (2003) who showed that $30 \%$ of transcripts are stage-specific. This, however, might be due to higher coverage of the transcriptomic data and technical issues with insoluble proteins in the proteome (Le Roch et al., 2006). Concurrent with the Florens et al. (2002) study, the proteome of the sexual developmental cycle of the parasite was delineated, which include 931 gametocyte and 645 gamete proteins amongst which were stage-specific, secreted, membrane-associated and conserved proteins (Lasonder et al., 2002). Some of these proteins contained cell-cell interaction domains that shed light on the biology of the sexual stages of the parasite and could be evaluated as components for transmissionblocking vaccines.

More focused studies were also conducted on subsections of the proteome. Novel GPIanchored proteins and their interacting partners were identified by analysis of 
compartmentalized proteomes of detergent resistant membranes of the malaria parasite (Sanders et al., 2005). Studies of infected erythrocyte membranes and Mauer's clefts of mature parasites led to the proposal that there is only a transient association of surface proteins with the Mauer's clefts ([Florens et al., 2004] and [Vincensini et al., 2005]). Similar studies were also performed on targeted organelles and life-cycle stages including the parasitophorous vacuole (Nyalwidhe et al., 2004), rhoptries (Sam-Yellow et al., 2004), and ookinete- and gametocyte-specific proteins (Khan et al., 2005). These studies highlighted the peculiarity of stage- and strategy-specific proteomes in addition to specialized proteomes of subcellular compartments in the parasite, all of which could be considered as potential parasite-specific drug targets. However, a high resolution proteomics investigation similar to the Bozdech et al. (2003) IDC transcriptome analysis is still lacking.

\section{Integrative biology from the Plasmodium transcriptome and proteome}

Very few reports nearing systems biological investigations of Plasmodia are available but biological questions regarding the parasitic lifestyle are starting to be addressed. The proteome data in combination with evidence of chromosomal clusters encoding coexpressed genes, suggest a highly coordinated expression of Plasmodium genes involved in common biological processes (Le Roch et al., 2004). There appears to be a positive correlation between the abundance of transcripts and their encoded proteins during the $P$. falciparum life-cycle ([Le Roch et al., 2006] and [Le Roch et al., 2004]). The majority of discrepancies are attributable to a delay between transcript production and protein accumulation due to a time-shift observed between detection of the transcript and its protein. This wide-spread phenomenon is localized to specific gene families. More interesting, however, are those transcripts/proteins that do not display the time-shift since they indicate controlling nodes in the physiology of the previous or the following stages of the parasite. For example, genes involved in cell cycle regulation in the liver schizont stage were frequently detected in the sporozoites and represent the parasites' ability to timeously adapt to the host environment during massive cell division in the liver (Le Roch et al., 2004). The transcript/protein accumulation discrepancy is also due to posttranscriptional regulation (including RNA stability and degradation) as well as genespecific control of translation or both. Although evidence of gene-specific control has been extensively shown for e.g. the var gene family, post-transcriptional regulation has been postulated to be the dominant mechanism of gene regulation in the P. falciparum IDC (Shock et al., 2007). A RNA helicase involved in mRNA decay appears to influence the stage-specific gene expression and regulation of the P. falciparum sexual cycle (Mair et al., 2006). Moreover, global genome-wide analysis indicated a progressive, stagedependent increase in decay rates during the IDC with RNA levels accumulating in the schizont stage (Shock et al., 2007). This stage-dependent genome-wide change in RNA decay rate seems currently to be a property unique to the malaria parasite. 
The most comprehensive and integrated analyses of the genome, transcriptome and proteome has been reported for $P$. berghei and $P$. chabaudi chabaudi and represents the state-of-the-art of functional genomics as applied to Plasmodia ([Fraunholz, 2005] and [Hall et al., 2005]). Hall and co-authors proposed that the parasite uses four strategies/mechanisms for gene expression during its lifecycle, which includes housekeeping, stage-specific, host-specific (mosquito or mammalian host) and strategyspecific genes (related to invasion, asexual replication and sexual development). Over half of the proteins were detected solely in one stage of the lifecycle, implying a considerable degree of specialization at the molecular level to support the demanding developmental program. A 47 nucleotide sequence motif occurs in two-thirds of the 3'UTRs of these transcripts, which implicated post-transcriptional gene silencing of gametocyte transcripts.

\section{The Plasmodium interactome}

No protein has physiological meaning alone and part of its mechanistic importance is determined by its interactions with other proteins and the cellular environment. An indepth understanding of the protein-protein interaction network (defined here as interactome) can provide valuable insights into the functions of proteins, the regulatory mechanisms employed by the parasite and their functional relationships (Box 1). An extensive protein-interaction study (nearing global-type analysis) using yeast-two hybrid technology was reported by LaCount et al. (2005). 2846 unique interactions were identified, which resulted in the grouping of previously uncharacterized proteins and the prediction of novel functions. The combination of this interaction information with coexpression data and gene ontology (GO) annotations, led the authors to identify groups of interacting proteins implicated in various biological processes including chromatin modification, transcription, mRNA stability, ubiquitination and host cell invasion. However, caution need to be exercised given the problems associated with yeast-two hybrid technologies including out-of-context interactions (e.g. compartementalization), structural artifacts and limited coverage. The experimental interactome dataset does however provide an opportunity for systems level analysis of the P. falciparum physiology through scale-free network comparisons and the identification of controlling protein-nodes, information which is still limited despite the availability of X-omic data. Comparison of the Plasmodium interactome with those of other organisms indicated a marked divergence with very little conservation with other protein complexes (Suthram et al., 2005). Only the endocytic complex, the unfolded protein response complex and the mini-chromosome maintenance (MCM) complex/heat shock response appear to be conserved when compared to the Saccharomyces interactome.

A genome-wide computational modeling of the P. falciparum interactome following integration of in silico and experimental functional genomics data, elucidated local and global functional relationships between gene products (Date and Stoeckert, 2006). Sixtynine different pathways, each with at least two components, were identified but 107 hypothetical proteins (out of 2216) were shown to interact solely with other hypothetical 
proteins, potentially representing new pathways or previously uncharacterized components of known pathways.

Comparative studies of protein network topologies and the controlling, highly connected nodes indicated an evolutionary conservancy and a well established inhomogeneity of biological networks, which highlighted the roles of controlling nodes. The P. falciparum interactome network was analyzed by combining experimental results ( $P$. falciparum interactome with genomic and proteomic data from other well-studied model organisms) and those of computational methods (Wuchty and Ipsaro, 2007). Functional clustering of the combined network of protein interactions revealed clusters of proteosomal, ribosomal and spliceosomal activities that were previously reported lacking in the experimental interactome dataset (Wuchty and Ipsaro, 2007). Moreover, the controlling nodes in the $P$. falciparum interactome was shown to feature an oligoarchy of highly interacting proteins indicating the presence of the so-called 'rich-club' phenomenon, where controlling nodes are well connected to each other (Wuchty, 2007). These results signifies a topological signature in the parasites' interactome, which covers parasite-specific biological features mainly revolving around invasion.

\section{In silico navigation of the Plasmodium X-omes}

One challenging and limiting factor of X-omic strategies lies in the analysis of the vast amounts of data generated. Various useful databases are available to the malaria community to translate data obtained by in silico mining of $\mathrm{X}$-omic data to biological meaning (Table 1) (Birkholtz et al., 2006b). The usefulness of in silico navigation and data mining of the transcriptome is illustrated by the similarity-based identification of 92 putative proteases in the $P$. falciparum genome (Wu et al., 2006) and members of the kinase family (Young et al., 2005).

Table 1.

A selection of tools available for the malaria community for the in silico analysis of $\mathrm{X}$ omic data

\begin{tabular}{|l|l|l|l|}
\hline Database & Site & Use & References \\
\hline PlasmoDB & http://www.plasmodb.org & $\begin{array}{l}\text { Plasmodium genome } \\
\text { resource }\end{array}$ & Bahl et al. (2003) \\
\hline $\begin{array}{l}\text { Malaria } \\
\text { Parasite } \\
\begin{array}{l}\text { Metabolic } \\
\text { Pathways }\end{array}\end{array}$ & http://sites.huji.ac.il/malaria & $\begin{array}{l}\text { Metabolic pathways of } \\
\text { malaria parasites, links to } \\
\text { PlasmoDB, GeneDB, } \\
\text { Brenda, Expasy }\end{array}$ & Ginsburg (2006) \\
\hline $\begin{array}{l}\text { DeRisi Lab } \\
\begin{array}{l}\text { Malaria } \\
\text { Transcriptome } \\
\text { Database }\end{array}\end{array}$ & http://malaria.ucsf.edu & $\begin{array}{l}\text { Transcript profiles during } \\
\text { IDC, several Plasmodium } \\
\text { strains }\end{array}$ & Bozdech et al. (2003) \\
\hline
\end{tabular}




\begin{tabular}{|c|c|c|c|}
\hline Database & Site & Use & References \\
\hline $\begin{array}{l}\text { Malaria IDC } \\
\text { Strain } \\
\text { Comparison } \\
\text { Database }\end{array}$ & $\begin{array}{l}\text { http://0- } \\
\text { malaria.ucsf.edu.innopac.up. } \\
\text { ac.za/comparison/index.php }\end{array}$ & $\begin{array}{l}\text { Transcript profiles during } \\
\text { IDC, several Plasmodium } \\
\text { strains }\end{array}$ & Llinás et al. (2006) \\
\hline GeneDB & $\begin{array}{l}\text { http://www.genedb.org/gene } \\
\text { db/malaria }\end{array}$ & General gene database & Sanger Institute \\
\hline BRENDA & $\begin{array}{l}\text { http://0-brenda.bc.uni- } \\
\text { koeln.de.innopac.up.ac.za/in } \\
\text { dex.php4 }\end{array}$ & $\begin{array}{l}\text { Enzyme characteristics } \\
\text { database }\end{array}$ & $\begin{array}{l}\text { Schomburg et al. } \\
(2002)\end{array}$ \\
\hline Expasy & http://au.expasy.org/ & $\begin{array}{l}\text { Expert Protein Analysis } \\
\text { System proteomics server }\end{array}$ & $\begin{array}{l}\text { Gasteiger et al. } \\
(2003)\end{array}$ \\
\hline Plasmo2D & $\begin{array}{l}\text { http://utlab3.biochem.iisc.ern } \\
\text { et.in/Plasmo2D/index.htm }\end{array}$ & $\begin{array}{l}\text { Plasmodium 2D-gel } \\
\text { electrophoresis proteomic } \\
\text { analysis }\end{array}$ & $\begin{array}{l}\text { Khachane et al. } \\
(2005)\end{array}$ \\
\hline FatiGO & http://fatigo.bioinfo.cipf.es/ & GeneOntology Mining & $\begin{array}{l}\text { Al-Shahrour et al. } \\
(2004)\end{array}$ \\
\hline GoMiner & $\begin{array}{l}\text { http://0- } \\
\text { discover.nci.nih.gov.innopac. } \\
\text { up.ac.za/gominer/ }\end{array}$ & $\begin{array}{l}\text { GeneOntology Mining, } \\
\text { metabolic pathway } \\
\text { interpretation }\end{array}$ & Zeeberg et al. (2003) \\
\hline DAVID & $\begin{array}{l}\text { http://0- } \\
\text { david.abcc.ncifcrf.gov.innop } \\
\text { ac.up.ac.za/ }\end{array}$ & $\begin{array}{l}\text { GeneOntology Mining, } \\
\text { metabolic pathway } \\
\text { interpretation }\end{array}$ & Dennis et al. (2003) \\
\hline GeneXPress & $\begin{array}{l}\text { http://0- } \\
\text { genexpress.stanford.edu.inno } \\
\text { pac.up.ac.za/ }\end{array}$ & $\begin{array}{l}\text { Metabolic pathways, } \\
\text { transcription regulation } \\
\text { visualisation }\end{array}$ & Segal et al. (2004) \\
\hline $\begin{array}{l}\text { The Gene } \\
\text { Ontology }\end{array}$ & $\begin{array}{l}\text { http://www.geneontology.org } \\
\text { /GO.tools.microarray.shtml }\end{array}$ & $\begin{array}{l}\text { Links to tools for gene } \\
\text { expression analysis }\end{array}$ & $\begin{array}{l}\text { Ashburner et al. } \\
(2000)\end{array}$ \\
\hline
\end{tabular}

The top half of the table indicates searchable databases and the bottom half, tools for the biological interpretation of $\mathrm{X}$-omic data

Any of the abovementioned X-omic strategies are potential starting points for the discovery of novel drug targets in $P$. falciparum, whether through new/additional functionality that is ascribed to a known protein or the characterization of novel function of a previously 'unknown' protein. The hypothetical proteins, that lack sequence 
similarity to genes from other known organisms, still represent more than half (

of the malarial proteome ([Birkholtz et al., 2006b] and [Gardner et al., 2002]) and signify some of the most attractive entities for selective targeting of the parasite. Guilt-byassociation (Oliver, 2000) strategies like ontology-based pattern identification (Zhou et al., 2005) attempts to assign functionality to genes (including hypotheticals) based on similar expression profiles, protein-interacting partners and subcellular organization. Application of these principles to the pentose-phosphate pathway and genes for enzymes in the antioxidant defense mechanisms of the parasite (Bozdech and Ginsburg, 2004) revealed a biphasic pattern in the transcription profile of several genes and that not all genes in this pathway are coordinately transcribed ([Bozdech and Ginsburg, 2004] and [Bozdech and Ginsburg, 2005]). However, Llinás and del Portillo cautioned against using only classical guilt-by-association methods, since many genes that are functionally unrelated show similar expression profiles during the asexual development phase of $P$. falciparum (Llinás and del Portillo, 2005). Examination of the spatial correlation between gene expression and chromosomal localization may be required to indicate co-regulation of genes. Identification of clusters of co-regulated genes from transcriptome data could aid the prediction of gene-regulatory regions in $P$. falciparum, an otherwise onerous task on sequence level alone given the extreme $\mathrm{A}+\mathrm{T}$ richness of the non-coding regions of this genome. An in silico search of the upstream regions of genes expressed during the sexual developmental cycle of the parasite identified a statistically overrepresented and novel palindromic sequence, TGTANNTACA, that was shown to bind nuclear factors (Young et al., 2006). The recent discovery of additional regulatory motifs in co-expressed gene clusters indicated that the parasite might rely on multiple $c i s$-regulatory elements to control the activity of promoters (Gunasekera et al., 2007a). The identification of such motifs and their protein binding partners provides unique therapeutic targets for the simultaneous prevention of expression of these co-regulated clusters of genes.

\section{Plasmodium $\mathrm{X}$-omes in drug target and therapeutics discovery}

\subsection{Understanding inhibitory compounds' MOA through transcriptomics}

Drug specific effects on the metabolism of targeted tissues or organisms include up- or downregulation of the protein target(s), the upregulation of detoxification pathways (cytotoxic responses) and the upregulation of alternative or compensatory pathways as reflected by changes in the transcriptome/proteome of the organism. The identification and characterization of such affected pathways aids the understanding of the MOA of an inhibitory compound by assessing its effect on metabolism, probing biological mechanisms and optimization of inhibitor properties. Additionally, it could also provide an indication of the target affected by the inhibitor in instances where this is unknown. The positive correlation between transcriptional response and drug challenge has been applied with success in the antibacterial field and provided reference compendia of expression profiles after drug challenge to gain insights into a drugs' MOA ([Butcher and Schreiber, 2005], [Freiberg and Brotz-Oesterhelt, 2005] and [Freiberg et al., 2004]). For example, a comprehensive dataset of 430 microarray profiles was generated by treating 
M. tuberculosis with 72 different drugs and growth-inhibitory compounds, which revealed signature expression profiles of agents with known MOA (Boshoff et al., 2004). This has now been widely accepted as a compendium approach to understand drug MOA ([Butcher and Schreiber, 2005] and [Freiberg et al., 2004]) and has been extended to the eukaryote, S. cerevisiae (Oliver, 2002).

Transcriptional profiling of drug-challenged malaria parasites has been limited to a few studies to date. A semi-global sequence-independent method, serial analysis of gene expression (SAGE), was used to study the transcriptional response of unsynchronized asexual parasites to a $6 \mathrm{~h}$ chloroquine treatment (Gunasekera et al., 2003). More than a hundred differential transcripts were thus identified, including several unknown open reading frames. Interestingly, only 11 transcripts were downregulated after the chloroquine treatment. Among the large number of upregulated transcripts, PfMDR1 was identified, thus confirming reports of the involvement of this multi-drug resistance gene in chloroquine resistance. This investigation indicated the existence of a feedback mechanism, signaling a transcriptional response to chloroquine. A subsequent follow-up report identified 600 drug responsive genes after treatment with chloroquine (Gunasekera et al., 2007b). Analysis of the abundance of transcripts for enzymes involved in folate biosynthesis in a gene-by-gene approach with quantitative reverse transcription PCR after pyrimethamine and sulfadoxine treatment however showed a uniform downregulation of transcripts with no feedback mechanism (Nirmalan et al., 2002). A high-density shortoligonucleotide array study (Affymetrix system) and proteome analysis indicated that more pronounced changes were induced at the protein level after challenging $P$.

falciparum with a choline analogue without significant transcriptional responses specific to the drug's MOA (Le Roch et al., 2006). In contrast, polyamine inhibition with cytostatic inhibitors with a time-course analysis on oligonucleotide arrays showed drugspecific differential up-regulation of transcripts including various metabolic responses to overcome the polyamine deficiency such as increased transcript levels for enzymes involved in methionine and polyamine metabolism ([Birkholtz et al., 2006a], [Clark et al., 2008] and [van Brummelen et al., in review]). Transcriptional responses to other environmental perturbations (including chloroquine) have also been observed (M. Llinás, unpublished data), indicating transcriptional feedback mechanisms that is independent of the normal transcriptional/post-transcriptional regulation of the parasite.

Clearly, more studies are needed to resolve whether the transcriptional response, or lack thereof, of Plasmodia to drug challenge is compound- or pathway-specific, or only apparent at the post-transcriptional or -translational level. Draghici et al. (2006) also made it clear that the inconsistencies between the various microarray platforms (in situ synthesized short oligos, longer oligos, spotted oligos or cDNA) are so high that it is almost impossible to currently compare results from different platforms.

It should be borne in mind that the parasite is undergoing considerable changes in morphology, metabolism and nuclear content as it proceeds through the $48 \mathrm{~h}$ erythrocytic cycle. In this regard several experimental constraints should be considered when transcriptional responses in the malaria parasite to drug challenge are investigated (Fig. 1). Time-course studies are essential and need to be performed on synchronous parasites 
to analyze the transcriptional changes during the ongoing asexual developmental cycle at the highest resolution possible (Bozdech et al., 2003). A reference pool setup should be included in the microarray design to allow comparisons between time-points in order to discriminate between primary and secondary effects caused by drug treatment (Fig. 1). However, a point-by-point comparison is often challenging due to the fact that the parasite growth might slow down or enter a quiescent state. Therefore, comparison of the time series to the expected IDC at that particular time point is also required to obtain an indication of relative changes (van Brummelen et al., in review). This will additionally provide: (1) a relative position during development that all expressions can be scaled to and (2) allow determinations of deviation (due to environmental perturbations) from the normal progressive growth changes. Furthermore, it is evident that we still know comparatively little of the biology of the parasite and the defined subcellular compartments and pathways of the vast numbers of hypothetical proteins. Computational analyses of transcriptome results against this background of incomplete knowledge can at most serve as a guideline to the encoded products of the transcripts and their underlying biological role.

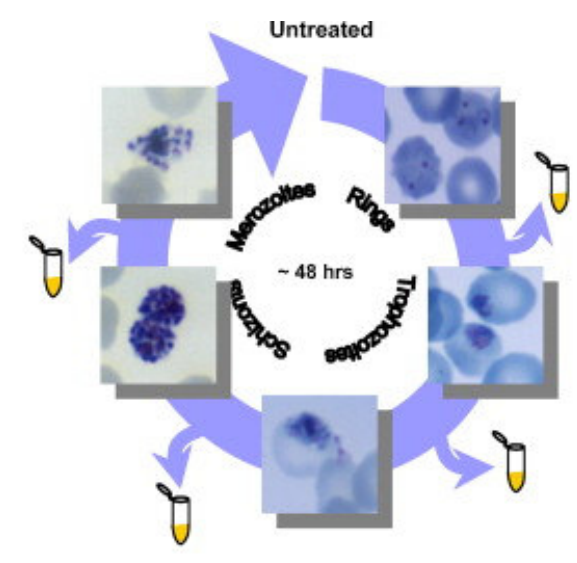

DNA microarray based Transcriptomics

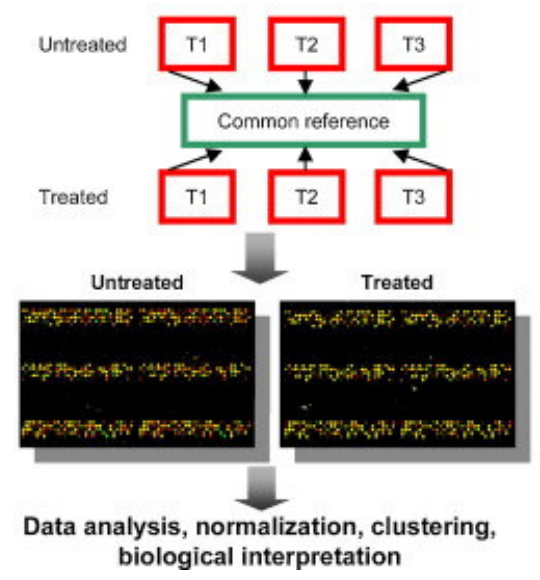

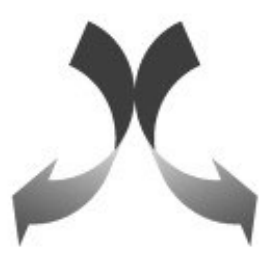

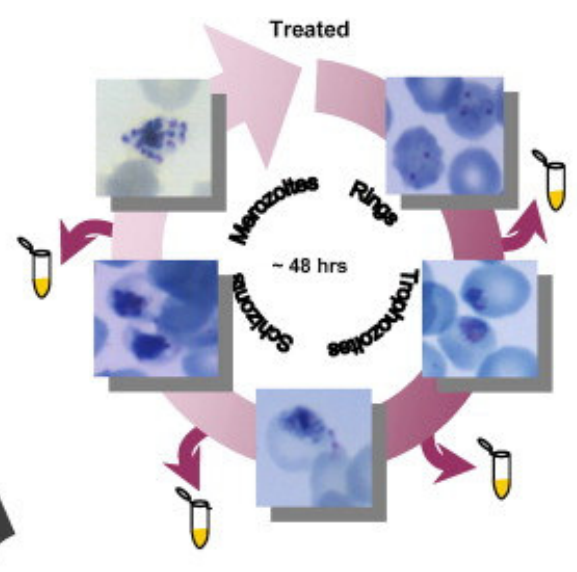

Quantitative Proteomics

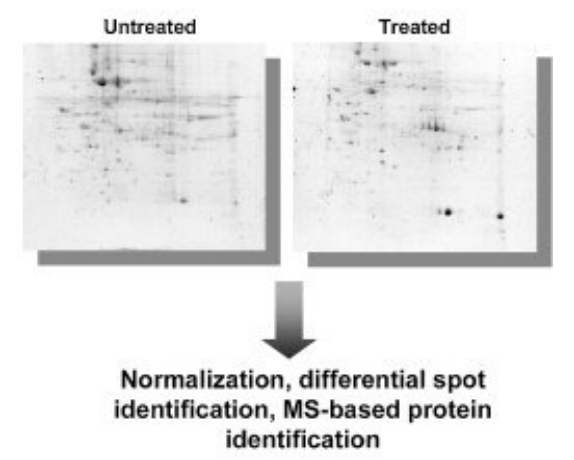


Fig. 1. Proposed experimental layout for the application of transcriptome and proteome analysis to drug challenged malaria parasites. Duplicate, synchronized P. falciparum asexual cultures should be maintained in parallel for untreated and drug treated cultures. Simultaneous RNA and protein isolation should be performed at the highest frequency possible (protein isolation will possibly have less time points due to technical constraints). DNA microarray performed on the long-oligonucleotide array (Bozdech et al., 2003) should have a reference design where the common reference pool (containing RNA starting material from all cultures at all time-points) is labeled with Cyanine 3 (green) and the treated/untreated time-point labeled with Cyanine 5 (red). This type of microarray design then allows a point-by-point comparison or a time course comparison to the IDC transcriptome. Changes in transcript levels can be compared to proteome changes with e.g. quantitative 2D-gel electrophoresis.

If the transcriptome analysis is to be extended to the proteome as well, it is advisable to isolate RNA and protein samples from the same cultures at the same time point to allow additional investigations of for example, post-transcriptional silencing. Apart from MudPIT, investigations of the proteome can be performed quantitatively with technologies including isotopically coded affinity tags (ICAT), isotope tags for relative and absolute quantification (iTRAQ) and quantitative 2D-gel electrophoresis ([Nirmalan et al., 2004a] and [Sims and Hyde, 2006]).

\subsection{Proteome-drug interactions}

Subproteome investigations have become particularly important to determine the molecular binding partner/target protein of an inhibitory compound and/or to describe the mode-of-action of chemical compounds. In this manner, proteins that bind ferriprotoporphyrin IX, which included glyceraldehyde-3-phosphate dehydrogenase, glutathione reductase and protein disulfide isomerase, were identified (Campanale et al., 2003). Several protein kinases that bind to known kinase inhibitors, could also be identified (Knockaert et al., 2000). Small synthetic molecules directed against different families of enzymes (e.g. proteases and phosphatases) have been developed as activitybased probes to complement chemical knockdown experiments and approaches in which novel inhibitors of the parasite are identified (Jeffery and Bogyo, 2004).

The most interesting example of such studies came from Graves and co-workers who applied affinity chromatography with quinoline drugs to screen the entire purine binding proteomes of $P$. falciparum and both mouse and human erythrocytes. Using this strategy, termed 'proteome mining', protein targets of the quinoline family were proposed to be only human proteins (aldehyde dehydrogenase family members e.g. aldehyde dehydrogenase 1 and quinone reductase 2 (Graves et al., 2002)). These results provide speculative views on the mode-of-action of these compounds.

Nirmalan et al. (2004b) followed their transcriptional response of antifolate inhibited parasites with investigations of the translational upregulation of these drug targets. In 
contrast with the changes in transcript levels of the drug target DHFR-TS, a marked increase was seen in protein levels upon treatment with inhibitors against this enzyme. This was specific to the inhibitor used and indicated that the parasite is able to significantly relieve translational constraints when faced with antifolate drug challenge. A recent study of proteome responses to the clinically used combination of artemether and lumefantrine indicated drug-specific changes in the proteome, including opposite effects by the two drugs on the expression of proteins involved in glycolysis but similar effects on stress-response proteins (Makanga et al., 2005).

\subsection{Interactome as drug target in malaria}

Due to the unique nature of the $P$. falciparum interactome, several possibilities present themselves in the discovery of mechanistically novel inhibitors. Importantly, essential proteins appear likely to have more interacting partners in interaction networks and the targeting of such proteins should have a more significant effect as functional nodes controlling entire networks (Apic et al., 2005). Advantages of targeting a specific pathway through protein-protein interactions include (1) greater structural variability in these interfaces, which can lead to more effective differentiation between parasite and host proteins (Archakov et al., 2003), and (2) slower development of resistance, since a functional mutation at both protein interfaces is necessary for effective resistance (Buendía-Orozco et al., 2005).

The exploitation of protein-protein interactions as antimalarial drug targets is enticing, especially in relation to functional nodes that control entire protein networks. Reports of modulators of protein-protein interactions being good drug targets are increasing (PerezMontfront et al., 2002) with examples including Trypanosoma brucei protein farnesyltransferase (Ohkanda et al., 2004) and P. falciparum triosephosphate isomerase (Singh et al., 2001). The inhibition of protein-protein interactions can be achieved by the use of synthetic peptides (peptidomimetics (Cochran, 2000)), bifunctional blockers with both highly specific and poorly reactive binding groups (Way, 2000) and new breakthroughs including potent 'credit-card' libraries of small, planar aromatic compounds that selectively bind to the interaction nucleating sites (Xu et al., 2006).

\subsection{Validation of drug targets}

Target validation is the process of linking drug targets with their therapeutic potential and confirming that a compound inhibits its intended protein target. Potential targets have to meet one requirement namely, they should generally be essential for growth of the organism. There are two main strategies in validation of drug targets: genetically, by showing that gene-disruption or loss-of-function of a protein results in nonviable or severely disabled organisms; or chemically, by demonstrating that a compound can selectively inhibit the function of a target protein resulting in cell death ([Cowman and Crabb, 2003] and [Wang, 1997]). The principle behind applying functional genomics to drug discovery is to validate potential drug targets by monitoring the global physiological effects of loss-of-function of the drug target on the phenotype of the organism at the transcriptome and/or proteome level (Wang et al., 2004, Williams, 2003). This could provide information on the underlying mechanisms caused by changes in linked networks that are controlled by overlapping functional nodes. In addition, it also allows the 
identification of secondary effects due to drug treatment in cases where multiple pathways in the cell are affected (Jackson and Phillips, 2002), or the identification of secondary effects caused by interruption of a protein network.

Parasitic protozoa and the Plasmodia in particular, are however not very amenable to classical genetic manipulation technologies resulting in the limited use of established strategies, including antisense and knock-out/knock-down technologies. The application of RNAi strategies in Plasmodia is a highly contentious issue since there is currently no evidence for significant homologs of the RNAi machinery in this organism (Brown and Catteruccia, 2006). The use of chemical inhibition and functional genomics-based techniques therefore becomes more valuable but conclusive proof-of-principle of smallmolecule based target validation for $P$. falciparum is not as evident as for other organisms ([Cowman and Crabb, 2003] and [Joachimiak et al., 2001]). A combination of chemical and genetic means is ultimately the best approach to prove the essential nature of a target (Jackson and Phillips, 2002).

It is evident from the above that the application of functional genomics for target validation in the malaria parasite is still in its infancy, but could prove a powerful technique worth exploiting if factors highlighted above are taken into consideration.

\section{Conclusions}

Several challenges face antiparasitic drug discovery. Potential targets should be genetically or chemically validated, biochemically and structurally analyzed, and should be amenable to high-throughput screening systems. In addition, therapeutics should be selective, economical and have good pharmacokinetic properties. These requirements are not unique to antimalarial drug discovery, but are more challenging since $P$. falciparum poses several technical constraints such as difficulties in obtaining sufficient quantities of recombinantly expressed protein for further downstream investigations. Functional genomics strategies have the ability to generate comprehensive information on the underlying biological network structure and dynamics of the interacting components of the malaria parasite as an integral whole. Such studies are expected to provide essential information to the malaria community in search of drug targets and new therapeutics. In particular, the fact that this organism is so extensively studied in terms of genomics over the majority of its life-cycle stages and with annotation of the majority of the predicted proteins still outstanding, these technologies provide unique opportunities to unlock biological properties of the parasite exploitable for therapeutic interventions.

The use and results of transcriptomic, proteomic and interactomic information were described here as well as their potential applications in drug discovery efforts. However, to complete this picture, extensive in vitro and in vivo data on e.g. metabolomics, glycomics, lipidomics are still lacking to approach a systems biology level of understanding of $P$. falciparum physiology. Major limitations facing us are mostly methodological in nature, including the availability of effective informatics tools for the mining and integration of data and experimental corroborations. 
The goals of systems biology include the discovery of interacting networks and their controlling nodes by integration of complex and diverse biological datasets into one system, either at the cellular or organism level. The interconnectivity of biological networks has been explored in various model systems including Saccharomyces, Haemophilus, Caenorhabditis, Drosophila and Mycobacterium and has led to e.g. the prediction of gene function and the elucidation of interacting networks (Winzeler, 2006). The challenge is therefore to translate these advances also to $P$. falciparum since we have by comparison only limited biological information of this protozoan parasite which has for centuries eluded the best efforts of humankind in controlling the disease.

\section{References}

Al-Shahrour et al., 2004 F. Al-Shahrour, R. Diaz-Uriarte and J. Dopazo, FatiGO: a web tool for finding significant associations of Gene Ontology terms with groups of genes, Bioinformatics 20 (2004), pp. 578-580.

Apic et al., 2005 G. Apic, T. Ignjatovic, S. Boyer and R.B. Russell, Illuminating drug discovery with biological pathways, FEBS Lett. 570 (2005), pp. 1872-1877.

Archakov et al., 2003 A.I. Archakov, V.M. Govorun, A.V. Dubanov, Y.D. Ivanov, A.V. Veselovsky, P. Lewi and P. Janssen, Protein-protein interactions as a target for drugs in proteomics, Proteomics 3 (2003), pp. 380-391.

Ashburner et al., 2000 M. Ashburner, C.A. Ball, J.A. Blake, D. Botstein, H. Butler, J.M. Cherry, A.P. Davis, K. Dolinski, S.S. Dwight, J.T. Eppig, M.A. Harris, D.P. Hill, L. Issel-Tarver, A. Kasarskis, S. Lewis, J.C. Matese, J.E. Richardson, M. Ringwald, G.M. Rubin and G. Sherlock, Gene ontology: tool for the unification of biology. The Gene Ontology Consortium, Nat. Genet. 25 (2000), pp. 25-29.

Bahl et al., 2003 A. Bahl, B. Brunk, J. Crabtree, M.J. Fraunholz, B. Gajria, G.R. Grant, H. Ginsburg, D. Gupta, J.C. Kissinger, P. Labo, L. Li, M.D. Mailman, A.J. Milgram, D.S. Pearson, D.S. Roos, J. Schug, C.J. Stoeckert and P. Whetzel, PlasmoDB: the Plasmodium genome resource. A database integrating experimental and computational data., Nucleic Acids Res. 31 (2003), pp. 212-215.

Birkholtz et al., 2006a L. Birkholtz, C. Claudel-Renard, K. Clark and A.I. Louw, Differential transcriptome profiling indicates the physiological significance of polyamines in the human malaria parasite Plasmodium falciparum, Keystone symposia: Malaria: Functional Genomics to Biology to Medicine (2006).

Birkholtz et al., 2006b L. Birkholtz, O. Bastien, G. Wells, D. Grando, F. Joubert, V. Kasam, M. Zimmerman, P. Ortet, N. Jacq, N. Saidani, S. Roy, M. Hormann-Apitius, V. Breton, A.I. Louw and E. Marechal, Integration and mining of malaria molecular, 
functional and pharmacological data: how far are we from a chemogenomic knowledge space, Malaria J. 5 (2006), p. 110.

Boshoff et al., 2004 H.I.M. Boshoff, T.G. Myers, B.R. Copp, M.R. McNeil, M. Wilson and C.E. Barry, The transcriptional response of Mycobacterium tuberculosis to inhibitors of metabolism, J. Biol. Chem. 279 (2004), pp. 40174-40184.

Bozdech et al., 2003 Z. Bozdech, M. Llinás, B.L. Pulliam, E.D. Wong, J. Zhu and J.L. DeRisi, The transcriptome of the intraerythrocytic developmental cycle of Plasmodium falciparum, PLoS Biol. 1 (1) (2003), p. e5.

Bozdech and Ginsburg, 2004 Z. Bozdech and H. Ginsburg, Antioxidant defence in Plasmodium falciparum: data mining of the transcriptome, Malaria J. 3 (2004), p. 23.

Bozdech and Ginsburg, 2005 Z. Bozdech and H. Ginsburg, Data mining of the transcriptome of Plasmodium falciparum: the pentose phosphate pathway and ancillary processes, Malaria J. 4 (2005), p. 17.

Brown and Catteruccia, 2006 A.E. Brown and F. Catteruccia, Toward silencing the burden of malaria: progress and prospects for RNAi-based approaches, Biotechniques 40 (2006), pp. s38-s44.

Buendía-Orozco et al., 2005 J. Buendía-Orozco, A. Guerrero and N. Pastor, Model of the TBP-TFIIB complex from Plasmodium falciparum: interface analysis and perspectives as a new target for antimalarial design, Arch. Med. Res. 36 (2005), pp. 317-330.

Butcher and Schreiber, 2005 R.A. Butcher and S.L. Schreiber, Using genome-wide transcriptional profiling to elucidate small-molecule mechanism, Curr. Opin. Chem. Biol. 9 (2005), pp. 25-30.

Campanale et al., 2003 N. Campanale, C. Nickel and C.A. Daubenberger, Identification and characterisation of heme-interacting proteins in the malaria parasite, Plasmodium falciparum, J. Biol. Chem. 278 (2003), pp. 27354-27361.

Clark et al., 2008 Clark et al., 2008. Biol. Chem. 389, in press.

Cochran, 2000 A.G. Cochran, Antagonists of protein-protein interactions, Chem. Biol. 7 (2000), pp. R85-R94.

Cowman and Crabb, 2003 A.F. Cowman and B.S. Crabb, Functional genomics: identifying drug targets for parasitic diseases, Trends Parasitol. 19 (2003), pp. 538-543.

Daily et al., 2004 J.P. Daily, K.G. Le Roch, O. Sarr, X. Fang, Y. Zhou, O. Ndir, S. Mboup, A. Sultan, E.A. Winzeler and D.F. Wirth, In vivo transcriptional profiling of Plasmodium falciparum, Malaria J. 3 (2004), pp. 30-37. 
Daily et al., 2005 J.P. Daily, K.G. Le Roch, O. Sarr, D. Ndlaye, A. Lukens, Y. Zhou, O. Ndir, S. Mboup, A. Sultan, E.A. Winzeler and D.F. Wirth, In vivo transcriptome of Plasmodium falciparum reveals overexpression of transcripts that encode surface proteins, J. Infect. Dis. 191 (2005), pp. 1196-1203.

Date and Stoeckert, 2006 S.V. Date and C.J. Stoeckert, Computational modeling of the Plasmodium falciparum interactome reveals protein function on a genome-wide scale, Genome Res. 16 (2006), pp. 542-549.

Dennis et al., 2003 G. Dennis Jr., B.T. Sherman, D.A. Hosack, J. Yang, W. Gao, H.C. Lane and R.A. Lempicki, DAVID: database for annotation, visualization, and integrated discovery, Genome Biol. 4 (2003), p. P3.

Draghici et al., 2006 S. Draghici, P. Khatri, A.C. Eklund and Z. Szallasi, Reliability and reproducibility issues in DNA microarray measurements, Trends Genet. 22 (2006), pp. 101-109.

Florens et al., 2002 L. Florens, M.P. Washburn, J.D. Raine, R.M. Anthony, M. Grainger, J.D. Haynes, J.K. Moch, N. Muster, J.B. Sacci, D.L. Tabb, A.A. Witney, D. Wolters, Y. Wu, M.J. Gardner, A.A. Holder, R.E. Sinden, J.R. Yates III and D.J. Carucci, A proteomic view of the Plasmodium falciparum life cycle, Nature 419 (2002), pp. 520526.

Florens et al., 2004 L. Florens, X.S. Liu, Y. Wang, S. Yang, O. Schwartz, M. Peglar, D.J.C. Yates III and J.R.Y. Wu, Proteomics approach reveals novel proteins on the surface of malaria-infected erythrocytes, Mol. Biochem. Parasitol. 135 (2004), pp. 1-11.

Fraunholz, 2005 M.J. Fraunholz, Systems biology in malaria research, Trends Parasitol. 21 (2005), pp. 393-395.

Freiberg et al., 2004 C. Freiberg, H. Brotz-Oesterhelt and H. Labischinski, The impact of transcriptome and proteome analyses on antibiotic drug discovery, Curr. Opin. Microbiol. 7 (2004), pp. 451-459.

Freiberg and Brotz-Oesterhelt, 2005 C. Freiberg and H. Brotz-Oesterhelt, Functional genomics in antibacterial drug discovery, Drug Disc. Today 10 (2005), pp. 927-935.

Gardner et al., 2002 M.J. Gardner, N. Hall, E. Fung, O. White, M. Berriman, R.W. Hyman and J.M. Carlton, Genome sequence of the human malaria parasite Plasmodium falciparum, Nature 419 (2002), pp. 498-511.

Gasteiger et al., 2003 E. Gasteiger, A. Gattiker, C. Hoogland, I. Ivanyi, R.D. Appel and A. Bairoch, ExPASy: the proteomics server for in-depth protein knowledge and analysis, Nucleic Acids Res. 31 (2003), pp. 3784-3788. 
Ginsburg, 2006 H. Ginsburg, Progress in in silico functional genomics: the malaria metabolic pathways database, Trends Parasitol. 22 (2006), pp. 238-240.

Gissot et al., 2004 M. Gissot, R. Phillip, S. Briquet, C. Boschet, S. Coupe, D. Mazier and C. Vaquero, Transcriptome of 3D7 and its gametocyte-less derivative F12 Plasmodium falciparum clones during erythrocytic development using a gene-specific microarray assigned to gene regulation, cell cycle and transcription factors, Gene 341 (2004), pp. 267-277.

Graves et al., 2002 P.R. Graves, J.J. Kwiek, P. Fadden, R. Ray, K. Hardeman, A.M. Coley, M. Foley and T.A.J. Haystead, Discovery of novel targets of quinoline drugs in the human purine binding proteome, Mol. Pharmacol. 62 (2002), pp. 1364-1372.

Guerra et al., 2006 C.A. Guerra, R.W. Snow and S.I. Hay, Mapping the global extent of malaria in 2005, Trends Parasitol. 22 (2006), pp. 353-358.

Gunasekera et al., 2003 A. Gunasekera, S. Patankar, J. Schug, G. Eisen and D. Wirth, Drug-induced alterations in gene expression of the asexual blood forms of Plasmodium falciparum, Mol. Microbiol. 50 (2003), pp. 1229-1239.

Gunasekera et al., 2007a A. Gunasekera, A. Myrick, K. Militello, J. Sims, C. Dong, T. Gierahn, K. Le Roch, E. Winzeler and D. Wirth, Regulatory motifs uncovered among gene expression clusters in Plasmodium falciparum, Mol. Biochem. Parasitol. 153 (2007), pp. 19-30.

Gunasekera et al., 2007b A.M. Gunasekera, A. Myrick, K.G. Le Roch, E.A. Winzeler and D.F. Wirth, Plasmodium falciparum: genome wide pertubations in transcripts profiles among mixed stage cultures after chloroquine treatment, Exp. Parasitol. 117 (2007), pp. 87-92.

Hall et al., 2005 N. Hall, M. Karras, J.D. Raine, J.M. Carlton, T.W. Kooij, M. Berriman, L. Florens, C.S. Janssen, A. Pain, G.K. Christophides, K. James, K. Rutherford, B. Harris, D. Harris, C. Churcher, M.A. Quail, D. Ormond, J. Doggett, H.E. Trueman, J. Mendoza, S.L. Bidwell, M.A. Rajandream, D.J. Carucci, J.R. Yates 3rd, F.C. Kafatos, C.J. Janse, B. Barrell, C.M. Turner, A.P. Waters and R.E. Sinden, A comprehensive survey of the Plasmodium life cycle by genomic, transcriptomic, and proteomic analyses, Science 307 (2005), pp. 82-86.

Jackson and Phillips, 2002 L.K. Jackson and M.A. Phillips, Target validation for drug discovery in parasitic organisms, Curr. Topics Med. Chem. 2 (2002), pp. 425-438.

Jeffery and Bogyo, 2004 D.A. Jeffery and M. Bogyo, Chemical proteomics and its applications to drug discovery, Drug Disc. Today 9 (2004), pp. S19-S26. 
Joachimiak et al., 2001 M.P. Joachimiak, C. Chang, P.J. Rosenthal and F.E. Cohen, The impact of whole genome sequence data on drug discovery-a malaria case study, $\mathrm{Mol}$. Med. 7 (2001), pp. 698-710.

Khachane et al., 2005 A. Khachane, R. Kumar, S. Jain, S. Jain, G. Banumathy, V. Singh, S. Nagpal and U. Tatu, Plasmo2D: an ancillary proteomic tool to aid identification of proteins from Plasmodium falciparum, J. Proteome Res. 4 (2005), pp. 2369-2374.

Khan et al., 2005 S.M. Khan, B. Franke-Fayard, G.R. Mair, E. Lasonder, C.J. Janse, M. Mann and A.P. Waters, Proteome analysis of separated male and female gametocytes reveals novel sex-specific Plasmodium biology, Cell (2005), pp. 675-687.

Knockaert et al., 2000 M. Knockaert, N. Gray and E. Damiens, Intracellular targets of cyclin-dependent kinase inhibitors: identification of affinity chromatography using immobilised inhibitors, Chem. Biol. 7 (2000), pp. 411-422.

LaCount et al., 2005 D.J. LaCount, M. Vignali, R. Chettier, A. Phansalkar, R. Bell, J.R. Hesselbert, L.W. Scheonfeld, I. Ota, S. Sahasrabudhe, C. Kurschner, S. Fields and R.E. Hughes, A protein interaction network of the malaria parasite Plasmodium falciparum, Nature 438 (2005), pp. 103-107.

Lasonder et al., 2002 E. Lasonder, Y. Ishihama, J.S. Andersen, A.M.W.P. Vermunt, A. Sauerwein, R.W. Eling, W. Hall, N. Waters, A.P. Stunnenberg and H.G.M Mann, Analysis of the Plasmodium falciparum proteome by high-accuracy mass spectometry, Nature 419 (2002), pp. 537-542.

Le Roch et al., 2003 K.G. Le Roch, Y. Zhou, P.L. Blair, M. Grainger, J.K. Moch, J.D. Haynes, P. De la Vega, A.A. Holder, S. Batalov, D.J. Carucci and E.A. Winzeler, Discovery of gene function by expression profiling of the malaria parasite life cycle, Science 301 (2003), pp. 1503-1508.

Le Roch et al., 2004 K.G. Le Roch, J.R. Johnson, L. Florens, Y. Zhou, A. Santrosyan, M. Grainger, F. Yan, K.C. Williamson, A.A. Holder, D.J. Carucci, J.R. Yates and E.A. Winzeler, Global analysis of transcript and protein levels across the Plasmodium falciparum life cycle, Genome Res. 14 (2004), pp. 2308-2318.

Le Roch et al., 2006 K.G. Le Roch, J.R. Johnson, H. Ahiboh, D. Plouffe, K. Henson, Y. Zhou, C. Mamoun, J.R.r. Yates, H. Vial and E.A. Winzeler, Genomic profiling of the malaria parasite response to the choline analogue reveals drug mechanism of action, Keystone Symposia: Malaria Functional Genomics to Biology to Medicine (2006).

Llinás and del Portillo, 2005 M. Llinás and H. del Portillo, Mining the malaria transcriptome, Trends Parasitol. 21 (2005), pp. 350-352. 
Llinás et al., 2006 M. Llinás, Z. Bozdech, E.D. Wong, A.T. Adai and J.L. DeRisi, Comparative whole genome transcriptome analysis of three Plasmodium falciparum strains, Nucleic Acids Res. 34 (2006), pp. 1166-1173.

Mair et al., 2006 G.R. Mair, J.A.M. Braks, L.S. Graver, J.C.A.G. Wiegant, N. Hall, R.W. Dirks, S.M. Khan, G. Dimopoulos, C.J. Janse and A.P Waters, Regulation of sexual development of Plasmodium by translational repression, Science 313 (2006), pp. 667669.

Makanga et al., 2005 M. Makanga, P.G. Bray, P. Horrocks and S.A. Ward, Towards a proteomic definition of CoArtem action in Plasmodium falciparum malaria, Proteomics 5 (2005), pp. 1849-1858.

Nirmalan et al., 2002 N. Nirmalan, P. Wang, P.F.G. Sims and J.E. Hyde, Transcriptional analysis of genes encoding enzymes of the folate pathway in the human malaria parasite Plasmodium falciparum, Mol. Microbiol. 46 (2002), pp. 179-190.

Nirmalan et al., 2004a N. Nirmalan, P.F.G. Sims and J.E. Hyde, Quantitative proteomics of the human malaria parasite Plasmodium falciparum and its application to studies of development and inhibition, Mol. Microbiol. 52 (2004), pp. 1187-1199.

Nirmalan et al., 2004b N. Nirmalan, P. Sims and J. Hyde, Translational up-regulation of antifolate drug targets in the human malaria parasite Plasmodium falciparum upon drug challenge, Mol. Biochem. Parasitol. 136 (2004), pp. 63-70.

Nyalwidhe et al., 2004 J. Nyalwidhe, S. Bauermeister and K. Lingelbach, Proteomic approaches towards the characterisation of the parasitophorous vacuole in Plasmodium falciparum-infected erythrocytes, Int. J. Med. Microbiol. (2004), pp. 29392-29393.

Ohkanda et al., 2004 J. Ohkanda, F.S. Buckner, J.W. Lockman, K. Yokoyama, D. Carrico, R. Eastman, K. de Luca-Fradley, W. Davies, S.L. Croft, W.C. Van Voorhis, M.H. Gelb, S.M. Sebti and A.D. Hamilton, Design and synthesis of peptidomimetic protein farnesyltransferase inhibitors as anti-Trypanosoma brucei agents, J. Med. Chem. 47 (2004), pp. 432-445.

Ohlstein et al., 2000 E.H. Ohlstein, R.R. Ruffolo Jr. and J.D. Elliot, Drug discovery in the next millennium, Annu. Rev. Pharmacol. Toxicol. 40 (2000), pp. 177-191.

Oliver, 2000 S. Oliver, Guilt-by-association goes global, Nature 403 (2000), pp. 601603.

Oliver, 2002 S.G. Oliver, Functional genomics: lessons from yeast, Phil. Trans. R. Soc. Lond. 357 (2002), pp. 17-23. 
Paolini et al., 2006 G.V. Paolini, R.H. Shapland, W.P. van Hoorn, J.S. Mason and A.L. Hopkins, Global mapping of pharmacological space, Nat. Biotechnol. 24 (2006), pp. 805-815.

Perez-Montfront et al., 2002 R. Perez-Montfront, M. Tuena de Gomez-Puyou and A. Gomez-Puyou, The interfaces of oligomeric protein as targets for drug design against enzymes from parasites, Curr. Topics Med. Chem. 2 (2002), pp. 457-470.

Pink et al., 2005 R. Pink, A. Hudson, M. Mouries and M. Bendig, Opportunities and challenges in antiparasitic drug discovery, Nat. Rev. Drug Disc. 4 (2005), pp. 727-740.

Ralph et al., 2005 S.A. Ralph, E. Bischoff, D. Mattei, O. Sismeiro, M. Dillies, G. Guigon, Y. Coppee, P.H. David and A. Sherf, Transcriptome analysis of antigenic variation in Plasmodium falciparum - var gene silencing is not dependent on antisense RNA, Genome Biol. 6 (2005), p. R93.

Sachs and Malaney, 2002 J. Sachs and P. Malaney, The economic and social burden of malaria, Nature 415 (2002), pp. 680-685.

Sam-Yellow et al., 2004 T.Y. Sam-Yellow, L. Florens and T.M. Wang, Proteome analysis of rhoptry-enriched fractions isolated from Plasmodium merozoites, J. Proteome Res. 3 (2004), pp. 995-1011.

Sanders et al., 2005 P.R. Sanders, P.R. Gilson, G.T. Cantin, D.C. Greenbaum, T. Nebl, D.J. Carucci, M.J. McConville, L. Schofield, A.N. Hodder, J.R. Yates III and B.S. Crabb, Distinct protein classes including novel merozoite surface antigens in raft like membranes of Plasmodium falciparum, J. Biol. Chem. 280 (2005), pp. 40159-40176.

Sawyer, 2006 T.K. Sawyer, Smart drug discovery leveraging innovative technologies and predictive knowledge, Nat. Chem. Biol. 2 (2006), pp. 646-648.

Schomburg et al., 2002 I. Schomburg, A. Chang and D. Schomburg, BRENDA, enzyme data and metabolic information, Nucleic Acids Res. 30 (2002), pp. 47-49.

Segal et al., 2004 E. Segal, R. Yelensky, A. Kaushal, T. Pham, A. Regev and D. Koller, GeneXPress: a visualization and statistical analysis tool for gene expression and sequence data, Eleventh Inter. Conf. on Intelligent Systems for Molecular Biology (2004).

Sexton et al., 2004 A.C. Sexton, R.T. Good, D.S. Hansen, M.C. D’Ombrais, L. Buckingham, K. Simpson and L. Schofield, Transcriptional profiling reveals suppressed erythropoiesis, up-regulated glycolysis and interferon-associated responses in murine malaria, J. Infect. Dis. 189 (2004), pp. 1245-1256.

Shock et al., 2007 J.L. Shock, K.F. Fishcer and J.L. DeRisi, Whole genomic analysis of mRNA decay in Plasmodium falciparum reveal a global lengthening of mRNA half-life during the intraerythrocytic development cycle, Genome Biol. 8 (2007), p. R134. 
Silvestrini et al., 2005 F. Silvestrini, Z. Bozdech, A. Lanfrancotti, E. Di Guilo, E. Bultrini, L. Picci, J.L. DeRisi, E. Pizzi and P. Alano, Genome-wide identification of genes upregulated at the onset of gametocytogenesis in Plasmodium falciparum, Mol. Biochem. Parasitol. 146 (2005), pp. 100-110.

Sims and Hyde, 2006 P.F.G. Sims and J.E. Hyde, Proteomics of the human malaria parasite Plasmodium falciparum, Expert Rev. Proteomics. 3 (2006), pp. 87-95.

Singh et al., 2001 S.K. Singh, K. Maithal, H. Balaram and P. Balaram, Synthetic peptides as inactivators of multimeric enzymes: inhibition of Plasmodium falciparum triosephosphate isomerase by interface peptides, FEBS Lett. 501 (2001), pp. 19-23.

Suthram et al., 2005 S. Suthram, T. Sittler and T. Ideker, The Plasmodium protein network diverges from those of other eukaryotes, Nature 438 (2005), pp. 108-112.

van Brummelen et al., in review van Brummelen et al., in review. Mol. Biochem. Parasit.

Vincensini et al., 2005 L. Vincensini, S. Richert, T. Blisnick, A. van Dorsselaer, E. Leize-Wagner, T. Rabilloud and C. Braun Breton, Proteomic analysis identifies novel proteins of the Mauers clefts, a secretory compartment delivering Plasmodium falciparum proteins to the surface of its host cell, Mol. Cell. Proteomics 4.4 (2005), pp. 582-593.

Wang, 1997 C.C. Wang, Validating targets for antiparasite chemotherapy, Parasitology 114 (1997), pp. S31-S44.

Wang et al., 2004 S. Wang, T.B. Sim, Y.-S. Kim and Y.-T. Chang, Tools for target identification and validation, Curr. Opin. Chem. Biol. 8 (2004), pp. 371-377.

Way, 2000 J.C. Way, Covalent modification as a strategy to block protein-protein interactions with small-molecule drugs, Curr. Opin. Chem. Biol. 4 (2000), pp. 40-46.

Williams, 2003 M. Williams, Target validation, Curr. Opin. Pharmacol. 3 (2003), pp. 571-577.

Winzeler, 2006 E.A. Winzeler, Applied systems biology and malaria, Nat. Rev. Microbiol. 4 (2006), pp. 145-151.

Wu et al., 2006 Y. Wu, X. Wang, X. Liu and Y. Wang, Data-mining approaches reveal hidden families of proteases in the genome of malaria parasites, Genome Biol. 13 (2006), pp. 601-616.

Wuchty, $2007 \mathrm{~S}$. Wuchty, Rich-club phenomenon in the interactome of $P$. falciparumartifact or signature of a parasitic life style, PloS ONE 3 (2007), p. e335. 
Wuchty and Ipsaro, 2007 S. Wuchty and J.J. Ipsaro, A draft of protein interactions in the malaria parasite P. falciparum, J. Proteome Res. 6 (2007), pp. 1461-1470.

Xu et al., 2006 Y. Xu, H. Lu, J.P. Kennedy, X. Yan, L.A. McAllister, N. Yamamoto, J.A. Moss, G.E. Boldt, S. Jiang and K.D. Janda, Evaluation of "credit card" libraries for inhibition of HIV-1 gp41 fusogenic core formation, J. Comb. Chem. 8 (2006), pp. 531539.

Ylostalo et al., 2005 J. Ylostalo, A.C. Randall, T.A. Myers, M. Metzger, D.J. Krogstad and F.B. Cogswell, Transcriptome profiles of host gene expression in a monkey model of human malaria, J. Infect. Dis. 191 (2005), pp. 400-409.

Young et al., 2006 J. Young, C. Benner, F. Yan, K. Chen, C. Glass, Y. Zhou and E.A. Winzeler, Malaria systems biology: elucidating mechanisms of Plasmodium falciparum transcriptional control, Keystone symposia: Malaria: Functional Genomics to Biology to Medicine (2006).

Young et al., 2005 J.A. Young, Q.L. Fivelman, P.L. Blair, P. de la Vega, K.G. Le Roch, Y. Zhou, D.J. Carucci, D.A. Baker and E.A. Winzeler, The Plasmodium falciparum sexual developmental transcriptome: a microarray analysis using ontology-based pattern identification, Mol. Biochem. Parasitol. 143 (2005), pp. 67-79.

Zeeberg et al., 2003 B.R. Zeeberg, W. Feng, G. Wang, M.D. Wang, A.T. Fojo, M. Sunshine, S. Narasimhan, D.W. Kane, W.C. Reinhold, S. Lababidi, K.J. Bussey, J. Riss, J.C. Barrett and J.N. Weinstein, GoMiner: a resource for biological interpretation of genomic and proteomic data, Genome Biol. 4 (2003), p. R28.

Zhou et al., 2005 Y. Zhou, J. Young, A. Santrosyan, K. Chen, F. Yan and E.A. Winzeler, In silico gene function prediction using ontology-based pattern identification,

Bioinformatics 21 (2005), pp. 1237-1245.

Corresponding author at: Department of Biochemistry, University of Pretoria, Pretoria 0002, South Africa. Tel.: +27 12420 2480; fax: +27 123625302 . 